\title{
Editorial. \\ Enhancing vaccine design strategies: \\ Applications for protein science, proteomics and adjuvants.
}

Yvonne Perrie, Helen R Griffiths and David Jones

Vaccines have saved millions of lives. Currently, and for infectious diseases in humans at least, sanitation and vaccination remains the most efficient and the most cost-effective prophylactic treatment available. Vaccination has led to the global eradiation of smallpox, the virtual eradication of polio and a reduction of over $95 \%$ in the incidence of diphtheria, tetanus, pertussis, measles, mumps and rubella [1]. Indeed, successful vaccine strategies have circumvented mortality caused by infectious diseases in developed countries and have thus increased our lifespan [1]. Today's society in developed countries has a life expectancy of more than 80 years and there is a higher proportion of elderly to young people. However, there remains a continued need for the development of new vaccines to offer protection for infections where we currently have no effective vaccine (infections such as malaria or HIV), and recurrent diseases (such as tuberculosis, respiratory syncytial virus, influenza $A$ and $B$ ), plus newly emergent diseases, such as West Nile fever or ebola. Vaccination is also increasingly recognised as an important strategy for food security, by providing protection to livestock against existing and emerging pathogens.

In developing vaccines, there is a range of options for consideration: 1) attenuated vaccines, where the infection is live but weakened e.g. BCG; 2 ) killed or in-activated vaccines, these are typically prepared by heat or chemical treatment and examples include influenza vaccines; 3 ) sub-unit or toxoid protein-based vaccines, e.g. HBV and 4) vaccines based on extended carbohydrate epitopes. These traditional approaches have proved successful, clearly evident by the reduction in mortality rates caused by of a number of infectious diseases. However, there can be numerous hurdles to consider in the selection of the vaccine platform design. This can include difficulty in cultivating the pathogen in vitro, as in the case with Hepatitis B virus (HBV) and parasites such as malaria and schistosoma. Determining that cultured pathogens are killed or sufficiently attenuated for safe inoculation is also of major concern. However, recombinant DNA technology has enabled the development of protein-based vaccines that can address these particular issues. Indeed, a recombinant protein of the HBV 
surface antigen, expressed by DNA-transfected cells, has led to a human vaccine against the virus. Other recombinant protein vaccines are being developed and tested including those for tuberculosis, herpes simplex virus (HSV-1), the influenza virus as well as cancers.

In recent times, prompted by several safety concerns, there has been a move away from whole-pathogen vaccines towards sub-unit and epitope ensemble vaccines. Sub-unit vaccines cannot revert to a virulent form; this is a phenomenon apparent amongst certain attenuated vaccines and can prompt a wide range of adverse effects, from severe morbidity to mortality. This can be supported further by the recent increase in knowledge and expertise in the field of biotechnology which opens up the ability to design new vaccines using synthetic peptides and proteins. Whilst these systems offer enhanced safety profiles compared to live attenuated systems, sub-unit vaccines tend to offer reduced potency. To address this issue adjuvant systems can be exploited.

To stimulate new collaborations and developments in vaccines, the British Council recently funded a workshop led by two organisations with complementary skills and objectives; Aston University in the UK, and Ho Chi Minh City Biotechnology Centre in Vietnam. This workshop brought together experience from protein science, biotechnology and pharmaceutical scientists to consider immuno-vaccinology and vaccine discovery and delivery strategies, using both informatics-based and experimental approaches. The workshop stimulated the collation of this special issue that highlights the current challenges faced in the development of new vaccines for human and animal welfare and outlines strategies for developing new vaccines.

Proteomic applications are providing new insights into vaccine development [2]. The review by Dunston et al explores how this technology can be exploited to (a) identify novel exofacial antigens, (b) provide detailed information on the molecular nature of any sub-unit vaccine and (c) provide insight into the host response to vaccination [2]. In another powerful application of proteomics, the paper by Munday et al explores how the syncytial viral infection of epithelial cells alters expression of mitochondrial proteins in a temporal manner [3]. While Tom was found to be protective, hsp90 supported epithelial cell infection by virus 
[3]. Such knowledge offers novel targets to explore in the development of anti-infective strategies.

Within this issue, we also explore the options available for the production of sub-unit vaccines using yeast. Yeast offers improved yield properties and the ability to synthesise more challenging antigens that we have struggled to produce in other systems [4]. Indeed Nguyen et al., demonstrate that $P$. pastoris yeast can express and secrete biofunctional fusion proteins [5]. Cuccui and Wren [6] have reviewed how glycosylation or the modification of a cellular component with a carbohydrate moiety e.g. bacterial $\mathrm{N}$-linked protein glycosylation can be exploited for glycobiotechnological applications including glycoconjugate vaccine and humanised glycoprotein production. The authors note that these conjugates may reduce the cost of vaccines thus offering further potential for glycoconjugate vaccines tailored specifically for animal vaccination [6]. McNair also highlights the need for improved animal health through vaccination [7]; drug resistance is prevalent in many ectoparasites (in human and domestic livestock) and new vaccines against such ectoparasites are urgently required. Similarly vaccines are required to protect animals against Mycoplasma mycoides-induced mastitis, pneumonia, arthritis, keratitis, as well as acute cases of septicaemia. In a review by Churchwood et al, six proteins were found to have less than $95 \%$ amino acid similarity to a closely related Mycoplasma species, thus demonstrating that they merit further investigation and evaluation in the development of diagnostic tests [8]. The challenge in developing effective canine infectious respiratory disease (CIRD) vaccines is also considered [9]; since CIRD is a complex syndrome involving a multifactorial etiology (including bacterial and viral pathogens) diagnosis and early treatment can be difficult and thus can affect the reduction in associated outbreaks. Vaccines are not available for some contributory or primary pathogens of CIRD and vaccination of animals during an outbreak may offer little advantage.

A range of methods are being explored to enhance the delivery and efficacy of vaccines. Adenoviral vectors are potent inducers of T-cell and antibody responses and Coughlan et al., review their pre-clinical and clinical development as novel vaccine vectors [10]. When considering the enhancement of these vaccines through delivery and adjuvants, Walters et al., [11] outline the potential for developing new delivery platforms that would provide singleshot vaccines (using encapsulation in biocompatible polymers) whilst Donnelly et al., [12] 
have looked at applying dissolving micro-needles as a delivery platform for vaccines. To boost adjuvanticity, Maughan et al., [13] have outlined new developments in the use of particulate inorganic adjuvants and Kastner et al., [14] have looked at the development of liposomal adjuvants using multivariate analysis.

To consider new methods for testing vaccines, Marshall et al., [15] have designed and characterised a multi-cellular model of human airways that takes into account the conditions in the airways and recapitulates many salient features, including the epithelial barrier and mucus secretion. This simulates the barriers to pulmonary delivery of particles and therefore represents a valid test platform for screening vaccines delivered via the pulmonary route.

In summary, whilst there are notable success stories in the development of vaccines, key challenges remain. To address these and develop the next generation of vaccines it is vital for us to exploit the full power of modern biotechnology and computer science as key tools for discovering new and commercially important immunogenic antigens. These must be combined with safe and effective adjuvants and delivery mechanisms to ensure that we can develop cost-effective vaccines that are globally accessible.

\section{Acknowledgements.}

The authors gratefully acknowledge the funding support from the British Council for funding the Researcher Links workshop 2013, 'Enhancing Vaccine Design and delivery strategies: Applications of protein science, proteomics and adjuvants'. 


\section{References:}

[1] Rappuoli, R., et al., Vaccines for the twenty-first century society. Nat Rev Immunol, 2011. 11(12): p. 865-72.

[2] Dunston C et al., JPP-14-0358

[3] Munday et al, Proteomic analysis of mitochondria in respiratory epithelial cells infected with human respiratory syncytial virus and functional implications for virus and cell biology. JPP-14-0325.R1

[4] Bill RM. Recombinant protein subunit vaccine synthesis in microbes: a role for yeast? 2015, special issue. JPP-14-0325.R1

[5]. Nguyen et al., Generation and characterization of soluble interleukin-33 receptor fused with immunoglobulin gamma-1 constant domain expressed by Pichia pastoris yeast. JPP-140322.R1

[6] Cuccui J and Wren B. Hijacking bacterial glycosylation for the production of glycoconjugates, from vaccines to humanised glycoproteins JPP-14-0327

[7] NcNair, CM. Ectoparasites of medical and veterinary importance: drug resistance and the need for alternative control methods. JPP-14-0312.R1

[8] Mitchell, The challenges in developing effective canine infectious respiratory disease (CIRD) vaccines. JPP-14-0337.R1.

[9] Churchward et al. Immunoproteomic characterisation of Mycoplasma mycoides subspeciescapri by mass spectrometry analysis of two-dimensional electrophoresis spots and western blot. JPP-14-0333.R1

[10] Coughlan et al. Adenoviral vectors as novel vaccines for influenza. JPP-14-0338.R1

[11] Walters et al. Next generation vaccines: single-dose encapsulated vaccines for improved global immunisation coverage and efficacy. JPP-14-0318.R1

[12] Maeliosa et al. Laser-engineered dissolving microneedle arrays for protein delivery: potential for enhanced intradermal vaccination JPP-13-0785

[13] Maughan et al. Particulate inorganic adjuvants: recent developments and future outlookJPP-14-0404.R1

[14] Kastner et al. Correlating liposomal adjuvant characteristics to in-vivo cell-mediated immunity using a novel Mycobacterium tuberculosis fusion protein: a multivariate analysis study DOI: $10.1111 /$ jphp. 12390.

[15] Marshall et al, Developing accurate models of the human airways. JPP-14-0391.R1 